\section{Laser Scanning Confocal Microscopy for Materials Science}

William McMillan

University of Newcastle, Australia

\section{Introduction}

Confocal microscopy has gained great popularity in biology and medical research because of the ability to image three-dimensional objects at greater resolution than conventional optical microscopes. In a typical Laser Scanning Confocal Microscope (LSCM), the specimen stage is stepped up or down to collect a series of two-dimensional images (or slices) at each focal plane. Conventional light microscopes create images with a depth of field, at high power, of 2 to $3 \mu \mathrm{m}$. The depth of field of confocal microscopes ranges from 0.5 to $1.5 \mu \mathrm{m}$, which allows information to be collected from a well defined optical section rather than from most of the specimen. Therefore, due to this "thin" focal plane, out of focus light is virtually eliminated which results in an increase in contrast, clarity and detection.

Coupled with a shallow depth of field, the confocal microscope also incorporates a pinhole in front of a photo-detector to only admit light that is in the same focal plane as the specimen. Again, this rejection of out of focus light allows true three-dimensional imaging of a specimen. The twodimensional slices that are collected can be reassembled to produce a three-dimensional data set, which can be manipulated to analyze and measure features of interest. Most commonly, the technique is applied to transmission optical microscopes for examining translucent objects. It can, however, be successfully applied to a standard metallurgical (reflected light) microscope, giving rise to an expanded set of applications. This gives a tool specifically designed for studying surfaces.

We are one of the few Universities in Australia using an LSCM for predominantly engineering and materials science applications. By exploring some examples, it is the aim of this article to give a general overview of how the LSCM can be used for materials science applications.

\section{General Applications}

Biological and medical applications of the confocal microscope have been very widely reported, and include imaging of large specimens such as insects; in-vivo imaging of tissue, skin lesions and cancers; labeling of botanical material, cells and neurons; imaging of bacteria, and even tadpole teeth.

Although not yet widely used in materials science, emerging applications include; imaging of paper fibers and coatings, study of corrosion products on surfaces, analysis of electronic circuit components (e.g., silicon chips), quantitative analysis of wear surfaces, grooves and cross sections, surface characterization of water droplets, emulsions and foams, investigation of depth and crack propagation, quantification of surface textures, flaws and features; determination of crystal topography; particle analysis and study of agglomeration, and even imaging sub-micron components (such as nanomachines, atomic force microscope probes, etc.).

The third major field of application is forensic science, which has a foot in both the biology and materials science camps. Examples include latent fingerprint detection, fluorescence imaging for counterfeit detection, fluorescent gels in DNA fingerprinting, three-dimensional imaging and comparison of footprints, indentation profiling of documents, examining hairs, textiles and fabrics, light filaments, soils, fertilizers, rifling and tooling marks on projectiles, embossing and embossed material, and much more.

\section{Specific Materials Applications}

The following "image gallery" consists of specimens that were imaged using our LSCM at the Department of Mechanical Engineering.

Figure 1, as follows, shows a cantilever and tip for an Atomic Force Microscope (AFM). The field of view is 192 by $192 \mu \mathrm{m}$. The AFM has similarities to conventional stylus profilometry, however the AFM can reveal a sample surface precisely to nanometer resolution in three dimensions. A variety of forces can be sensed by the AFM. In the non-contact mode (distances greater than 10 Angstrorns between the tip and sample surface), Van der Waals, electrostatic, magnetic or capillary forces produce images of topography, whereas in the contact mode, ionic repulsion forces take the leading role. The tip is mounted on a flexible beam (cantilever) whose geometrical and material properties make it possible to probe the force with a high sensitivity. The interaction of the tip with the force is sensed by the resultant deflection of the cantilever on which the tip is mounted. The forces acting on the the cantilever include its weight (a distributed force), the weight of the tip attached to its free end (a concentrated force) and a driving force acting on the cantilever (which acts as a concentrated force) $)^{1}$. The cantilever and tip shown in Figure 1 were used to determine the frictional forces between dry particles. From the three-dimensional data set it was possible to obtain the measurements required to calculate the deflection forces encountered by the probe and thereby analyze their subsequent effect on the results.

Figure 2, as follows, shows the surface of a glass fiber reinforced polymer composite material that has been exposed to a flow of hard particles. The field of view is 120 by $120 \mu \mathrm{m}$. The figure indicates the degree of abrasive wear inflicted on the surface, showing crushed and splintered fibers. This material was studied as an alternative to steel for use in the construction of train carriages for transporting coal. The wear test used simulated conditions of coal loading. Polymer composite products are becoming more and more widely utilized in industrial applications, replacing traditional engineering materials. The mechanical properties of glass re-enforced polymer composites, such as specific strength

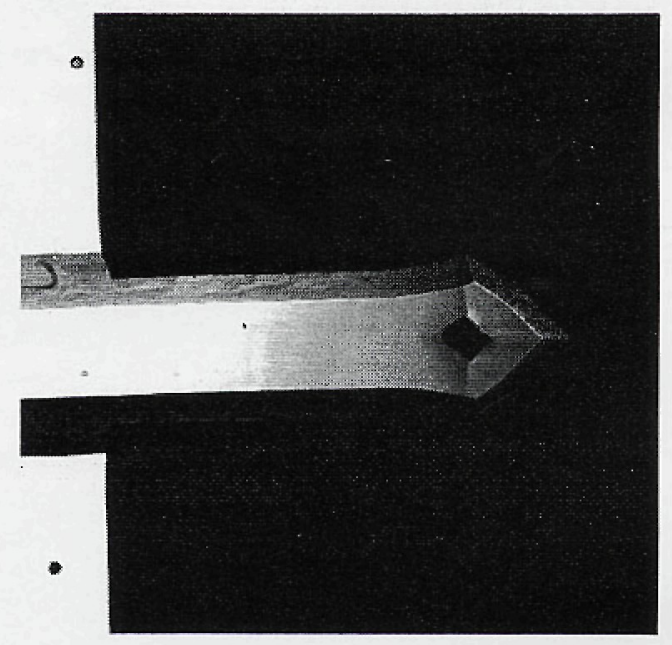

Figure 1 AFM cantilever and tip (192 x $192 \mu \mathrm{m})$.

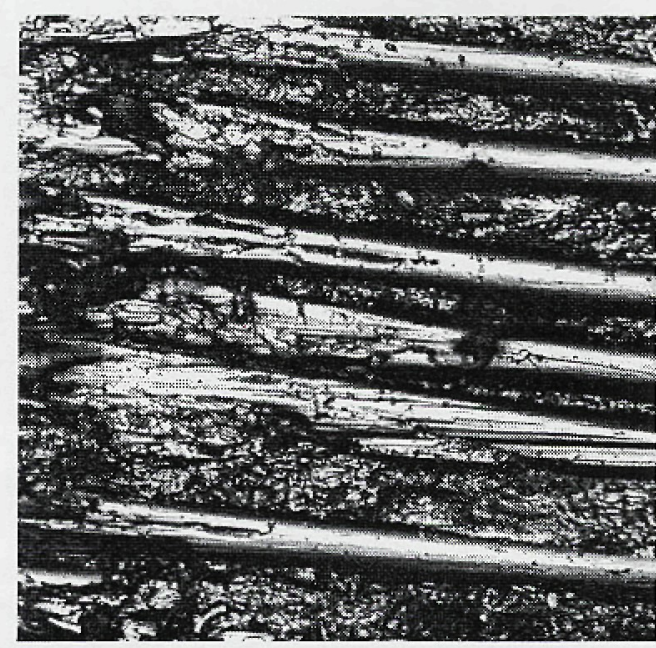

Figure 2 Wear surface of a glass fiber reinforced polymer composite material $(120 \times 120 \mu \mathrm{m})$. Supplied by A Goninan \& Co. Ltd. 
and wear resistance, are influenced by a number of factors, including the nature of the polymer matrix, the nature and diameter of the reinforcing fibers and the interfacial adhesion between the two. Confocal microscopy is useful for characterizing surface wear particularly in three dimensions. In the case of Figure 2, the LSCM can be used to determine which wear mechanism is acting on the surface, distinguishing between micro-ploughing, micro-cutting and micro-cracking. Figure 2 shows micro-cracking (the removal of material by fracturing particles out of

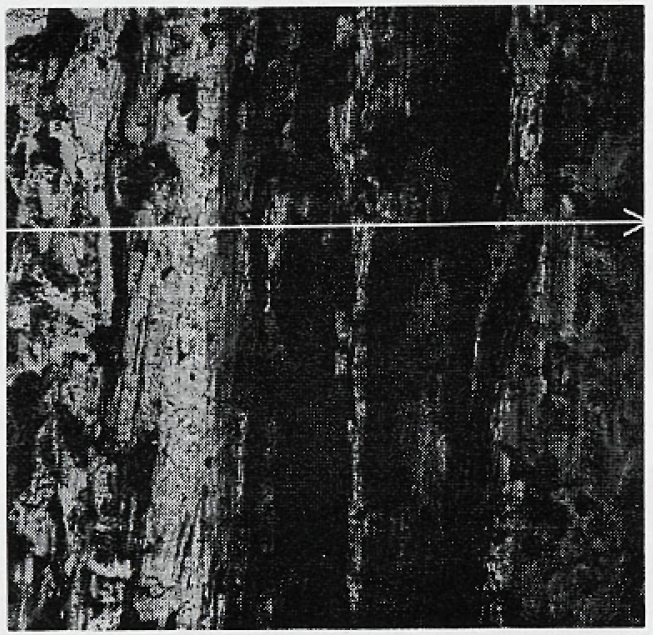

A

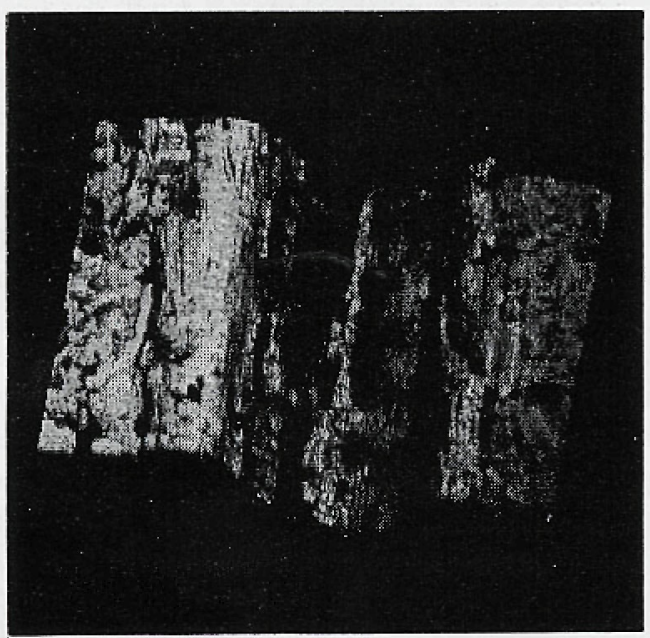

B

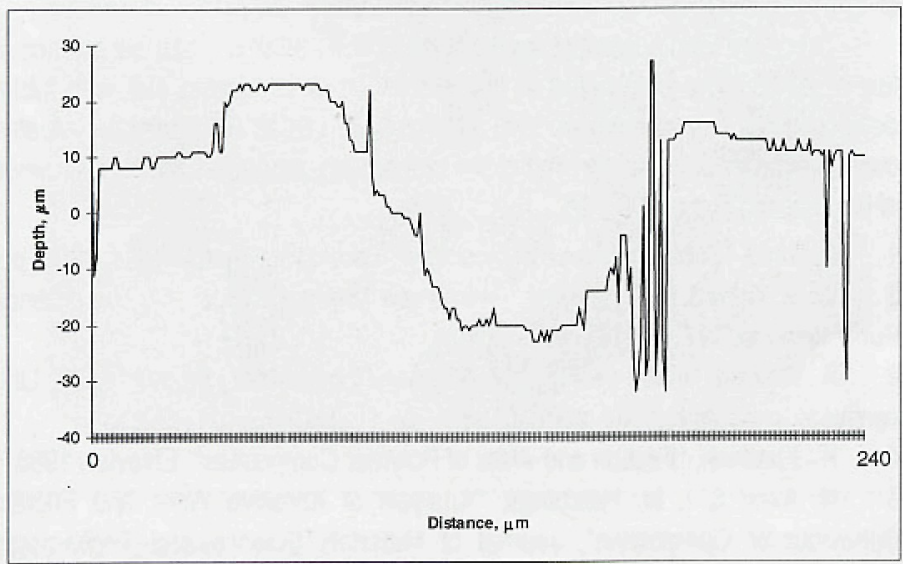

$\mathrm{C}$

Figure 3 (a) Wear groove resulting from metal to metal sliding contact ( $240 \times 240$ $\mu \mathrm{m})$, (b) three-dimensional representation of the wear groove and, (c) a profile across the groove, corresponding to the line in (a). the surface), together with micro-cutting (the removal of angular "chips" and the formation of scratch-like grooves) $)^{2-5}$. It is also useful for examining the interfacial bond between the matrix and fiber.

The information we can garner from images of wear surfaces include the calculation of wear volume and characterization of wear modes. It is possible to use this information to rank the wear characteristics and rates of different materials under similar testing conditions. It can also be useful to evaluate the effect of lubricants on the wear of certain materials.

The analysis of wear particles is widely applied for on-line condition monitoring of machinery, providing useful information for machine maintenance. Traditionally, visual analysis has often been time consuming, expensive and inconsistent, but with the application and further development of the LSCM, three-dimensional images of wear particles can be obtained ${ }^{6}$.

The advantage of confocal microscopy is the fact that we can use the three-dimensionality of the data to profile surfaces and obtain quantitative data. Optical profiling is more flexible than conventional stylus profilometry as there are no geometry constraints involved, and there is no mechanical contact between $\subsetneq$ the sample surface and stylus, which could have a damaging effect on delicate surfaces. Figure 3 , as follows, shows a wear groove in two dimensions, obtained by metal-metal sliding contact, a three-dimensional representation of the same $\bar{\Phi}$ groove, and the resultant groove profile, which provides us with information regarding the degree of plastic deformation at the sides of the groove, as well as

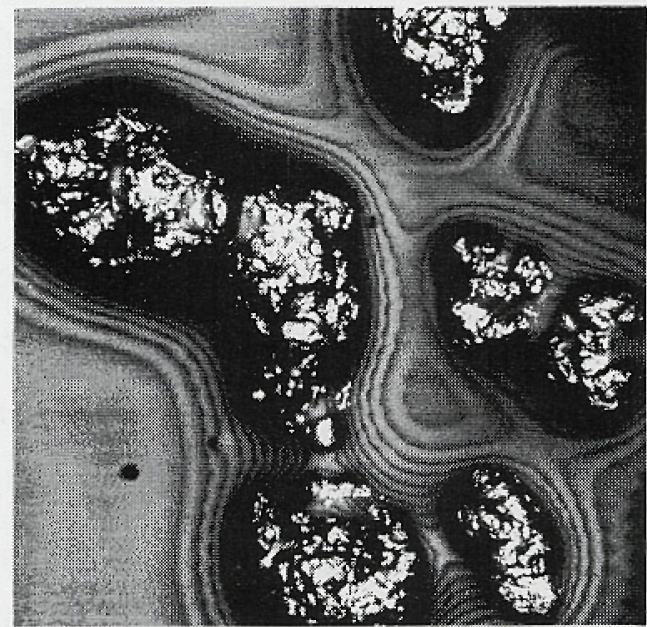

A

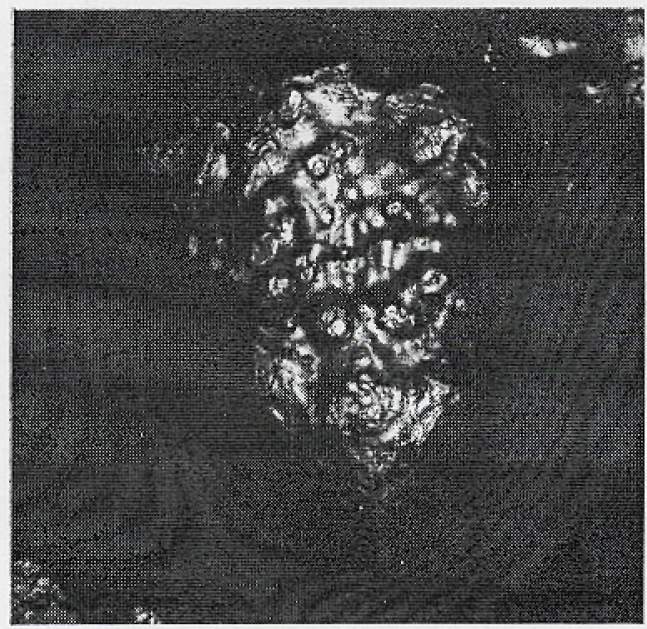

B

Figure 4 (a) Dispersion of small iron particles $(240 \times 240 \mu \mathrm{m})$ and, $\mathrm{m}$ (b) closer view of an iron particle showing surface details $(120 \times 120 \mu \mathrm{m})$

Continued on page 22 


\section{Laser Scanning Confocal Microscopy for Material Science - Continued from page 21}

the depth and cross-sectional area of the groove. As with the wear of the glass fiber reinforced composite material outlined above, LSCM can be used to image and thence characterize the various wear mechanisms that are acting for any set of wear conditions.

Analysis and distribution of particles is an important application for confocal microscopy in materials science. Figure 4 shows an overview of acid washed iron particles ( 240 by $240 \mu \mathrm{m})$ and a closer view of one of the particles, detailing the surface features $(120$ by $120 \mu \mathrm{m})$. There is considerable surface detail for a particle that is less than one-tenth of a millimeter in diameter. Figure 4 helps to illustrate the image quality that can be achieved by the LSCM.

The final application for discussion is the use of confocal microscopy to image emulsions. Figure 5 is an emulsion used in the manufacture of certain explosives. The field of view is 300 by $300 \mu \mathrm{m}$. From this image it is possible to calculate the droplet size and shape distribution, and from the threedimensional data set, we can optically profile the surface. Profiling by conventional stylus techniques is impossible due to the nature of the emulsion, thus non-contact profilometry is the only option.

Emulsions, such as those in Figure 5, have time-dependent properties. We can use the LSCM to take images at predetermined time intervals in order to capture the progress of a certain chemical reaction. This function is most useful when adding foaming reagents to emulsions, whereby an almost spontaneous initial effect can be observed, followed by a gradual reduction in the rate of the reaction, until stability is reached. The LSCM can be used to monitor changes and the gradual differences in the rate of reaction over time.

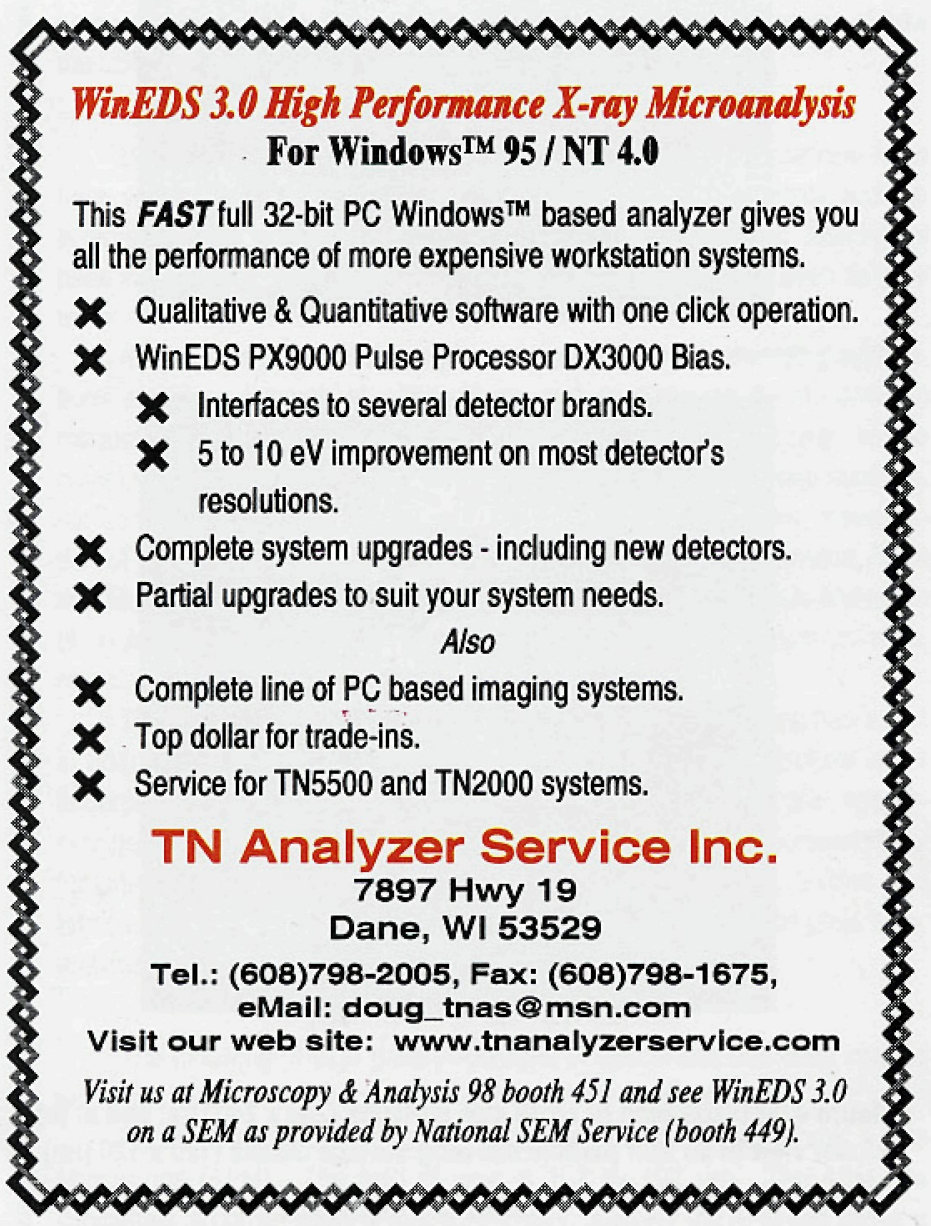

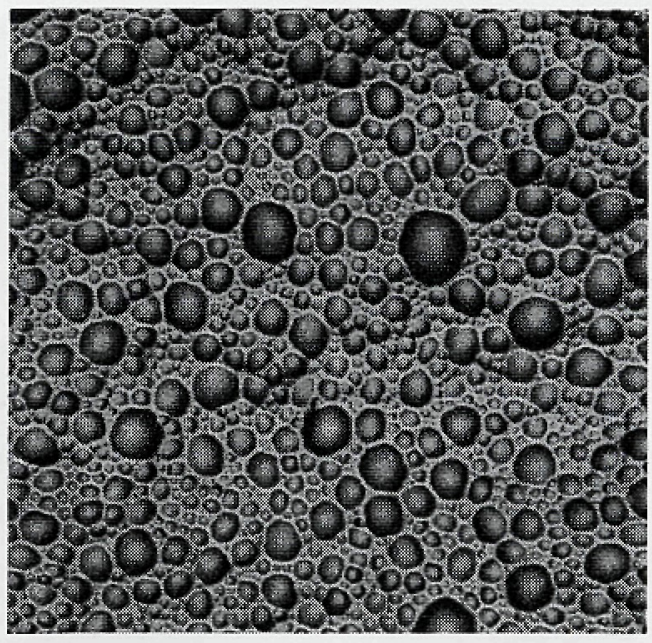

Figure 5 Droplet distribution in an emulsion used for explosives applications $(300 \times 300 \mu \mathrm{m})$

\section{Discussion}

Laser scanning confocal microscopy is beginning to find many new and exciting applications in materials science and engineering. From the few examples outlined in this article it can be seen that a diverse range of images and quantitative information can be obtained from this technique. It is believed LSCM will continue to grow in popularity for non-biological applications, as surface technology, tribology, interfacial bonding and advanced materials characterization are becoming increasingly critical in industrial applications.

The image quality from LSCM, in terms of resolvable detail, is comparable to scanning electron microscopy, though the LSCM cannot achieve the same magnification nor can it perform elemental analysis. However, LSCM has advantages in that specimen preparation is the same as for standard optical techniques, and imaging is conducted under atmospheric conditions. The sample is only limited in the fact that it has to be able to fit onto a standard microscope stage. With the emergence of endoscope type confocal systems, even this limitation should be short lived.

Conventional light microscopy provides information in only two dimensions, which means that a lot of information regarding the topography and morphology of a surface is superimposed. With the three-dimensional capability of laser scanning confocal microscopy, this information can be gathered to give "an extra dimension" of quantifiable data. The resultant optical profiling is more accurate, non-destructive and not subject to geometric constraints compared to conventional stylus profilometry, which has a geometric constraint and contact application. With optical profilometry we are only constrained by the wavelength of light we are using. The wavelength of light used in our system is $488 \mathrm{~nm}$.

There are many potential applications for the LSCM in materials science, a few of which were canvassed in this article. It is envisaged that with future developments like endoscopy and polarization, LSCM will make a valuable contribution to our understanding of the processing, properties and performance of engineering materials.

1 D. Sarid, "Scanning Force Microscopy", Oxford University Press, 1994, p1 2 W. V. Titow \& B. J. Lanham, "Reinforced Thermoplastics", Applied Science Publishers Ltd, 1975, pp182-185

3 A. Whitten, "Abrasive Wear of Polymer Composites", Project report, University of Newcastle, 1997, pp6-10, p50

4 K. Friedrich, "Friction and Wear of Polymer Composites", Elsevier, 1986

5 N. Axen \& I. M. Hutchings, "Analysis of Abrasive Wear and Friction Behaviour of Composites", Journal of Materials Science and Technology, Vol.12, September 1996, p757

$6 \quad$ Z. Peng, T. B. Kirk \& Z. L. Xu, "The Development of Three-Dimensional Imaging Techniques of Wear Particle Analysis", Wear, Vol.203-204, 1997, pp418-424 


\section{FTHIR}
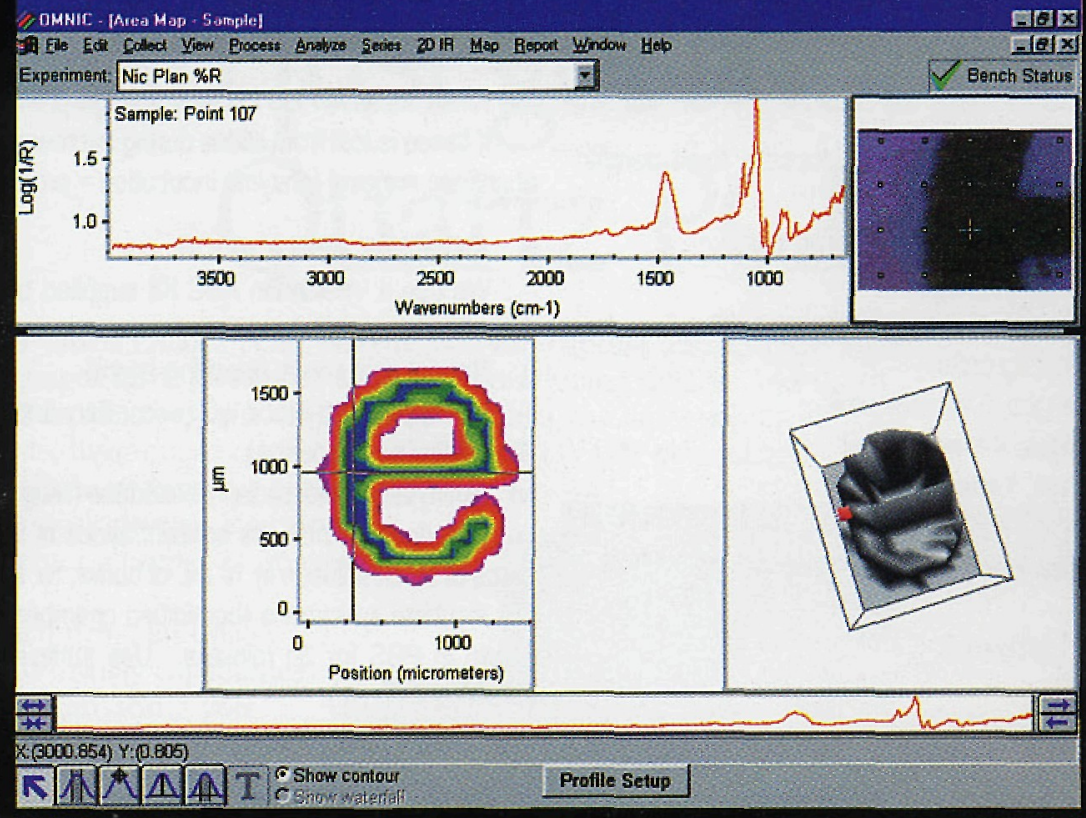

\section{Results Focused}

Nicolet understands the modern laboratory is a busy place. That is why we have added our unique E.S.P. (Enbanced

Synchronization Protocol) technology to every system, allowing Nicolet spectrometers to complete many tasks without assistance. This leaves you free to concentrate on your work.

Take a Nicolet microscopy system, for example. Using OMNIC ${ }^{\infty}$ Atl $\mu s^{\text {Th }}$ automated microscope and mapping software, simply place your sample in the microscope and you are ready to go. Point and click interaction means you can analyze your data with ease. Atlus software even updates all of the views on your screen (spectrum, video image, contour and

3D maps) to correspond to the area you are interested in, giving you complete perspective.

If you are focused on results, Nicolet gets you there.

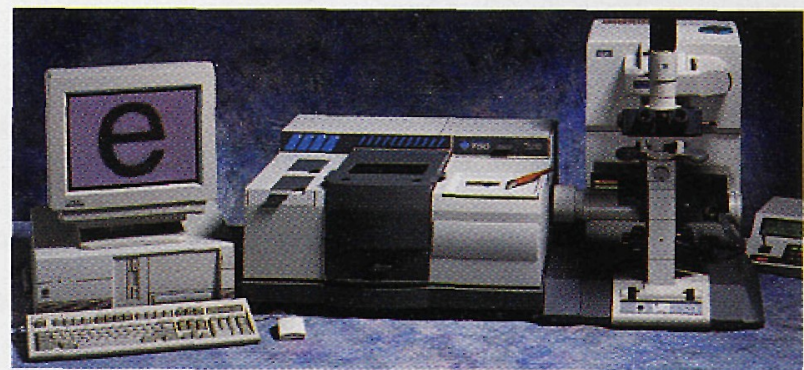

Magna-IR ${ }^{2} 760$ E.S.P. Spectrometer with Nic-Plan ${ }^{\text {Tw }}$ Microscope
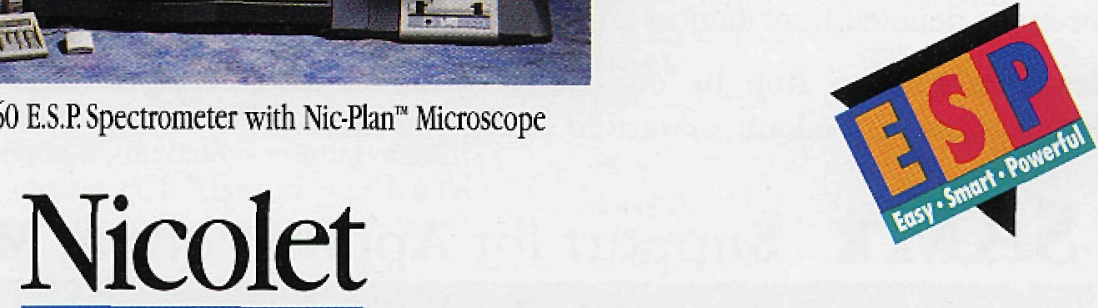

INSTRUMENTS OF DISCOVERY

A subsidiary of Thermo Optck Corporaton, a Thermo Instrument Systems, Inc. company 